\title{
Abstract \\ Mmoving beyond routine antenatal exercises: Incorporating mindful movement into antenatal care in Sri Lanka
}

\author{
Pattiyakumbura $\mathrm{T}^{1^{*}}$, Agampodi $\mathrm{SB}^{1}$, Agampodi $\mathrm{TC}^{1}$, Katumuluwa $\mathrm{S}^{1}$, Rankaduwa $\mathrm{NP}^{1}$ \\ ${ }^{1}$ Department of Community Medicine, Faculty of Medicine and Allied Sciences, Rajarata University of Sri \\ Lanka
}

\begin{abstract}
Background

There is evidence to suggest that physical activity during pregnancy may have many benefits to the pregnant mother and infant, both during the pre- and postnatal periods. Our program aims to eventually incorporate mindful movement into antenatal clinics in Sri Lanka to promote physical and mental well-being.

\section{Methods}

We conducted a pilot feasibility study to provide training in mindfulness to 12 pregnant women in Nuwaragam Palatha Central, Anuradhapura. Mindfulness has been defined as "paying attention in a particular way; on purpose, in the present moment, and non-judgmentally." A main component of our program is mindful movement, which consists of a series of prenatal yoga poses, a gentle movement series (stretches), and walking meditation. After practicing all the mindful movement activities, participants were asked for their feedback through an anonymous, semi-structured, self-administered questionnaire to evaluate their acceptability and possible benefits to the pregnant population.

\section{Results}

Most women reported feeling a calmness of their body and mind, stress reduction, and better ability to concentrate while performing the techniques. They also reported experiencing better sleep, relief from body pain/discomfort, and a greater ability to keep their mind in the present moment after practicing the techniques for several weeks on their own. Several had also involved their children in the activities. One woman suggested dedicating more time to mindful movement in the training. All women felt that incorporating mindful movement training into the national antenatal care system would be beneficial to pregnant women and their families.

\section{Conclusions}

Based on feedback from participants, mindful movement techniques would be a culturally acceptable and beneficial approach to encourage women to continue to be physically active during pregnancy.
\end{abstract}

Key words: Mindful Movement; Pregnancy; Antenatal Clinics

Copyright: (C) 2015 Pattiyakumbura $\mathrm{T}$ et al. This is an open access article distributed under the Creative Commons Attribution License, which permits unrestricted use, distribution, and reproduction in any medium, provided the original work is properly cited.

* Correspondence : thulanipattiyakumbura@yahoo.com

Cite this abstract as: Pattiyakumbura T, Agampodi SB, Agampodi TC, Katumuluwa S, Rankaduwa NP. Moving beyond routine antenatal exercises: Incorporating mindful movement into antenatal care in Sri Lanka. Anuradhapura Medical Journal 2015;9 (2Supp):S30.

DOI: http://dx.doi.org/10.4038/amj.v9i2Supp.7579 
